\title{
The Excellent Method of Novel Uncertainty Predictor for Risk Assessment in E-Financial Institutions
}

\author{
Alireza Miremadi and Omidreza Ghanadiof
}

\begin{abstract}
Financial institutions are always engaged in risk assessment. In fact, the birth of finance in Europe was through dealing with risk. Risk is a situation that we have more than a possible future with different probabilities. Assessing different futures with different level of probabilities is not that easy to formulate. Neural network is a topology developed for dealing with cases in which formulating the problem is not easy due to model flexibility which is required by the conditions of risks we are dealing with in E-finance. E-finance is providing financial services over electronic devices and cyber space. With the prominent growth of E-finance, the need for developing new models for assessing risk associated with this kind of business seems inevitable. Continuous growth of $E$-finance brings on new issues such as E-trust; consequently, the need for developing a model of total risk assessment is the base of our study. The presented model is a prototype, future models should be developed specifically for different kind of risk E-finance provider are dealing with.
\end{abstract}

Keywords - Back propagation, E-Finance, E-Trust, Neural Networks, Predictive Controller, Risk Assessment.

\section{INTRODUCTION}

From the very first moment decisions in all aspect of our life are associated with an element of uncertainty and probability. There are many different approaches to define [1], [2] risk, but all approaches share the view that risk is caused by uncertainty or better stated risk is a situation where we are dealing with more than one outcome and desirability of the outcomes are genuinely different. In these kinds of situations usually we face a loss/gain potential. Any situation containing more than one future is a risky situation.

Financial sector from its very first origin is based on risk. The aim of all players in this ground from the very beginning was - and is - to maximize ones benefit through good strategies for dealing with risky situation. Banking and insurance, from the very beginning of their construction were dealing with uncertain terms. Considering the traditional marine insurance, back in $15^{\text {th }}$ century, as one of the very first and pioneering fields of risk and financing studies, to the current option and security markets, people are taking chances and studying the outcome of risk as a mean to understand how they should deal with this wavy and forceful sea in order to gain benefit from it.

Introduction of electronic devices and the way they have changed our life and perceptions is a revolution by all means. But the second wave of this revolution, cyber space, and

Published on July 28, 2021.

Dr. Alireza Miremadi, Dean \& Assistant Marketing Professor at Sharif University of Technology-International Campus, Iran.

(e-mail: ar.miremadi@sharif.edu /alireza.miremadi@gmail.com) introduction of web2 and cloud computing, are just the vanguard for next generation of change resulting in the need for developing new techniques and models for assessing the situations we would be dealing with.

Electronic finance is the transition of traditionally physical services into electronic devices. From the introduction of NAZDAQ in 1979, the electronics has rocked the financial world non-stop [3]. Now a day all kind of financial transaction and operations are dealt with help of electronic devices. People are using cell phones as a replacement of plastic cards, even though plastic payment card themselves were electronically enhanced services provided by financial sector. But it is not just the payment which is becoming online worldwide.

With the help of E-finance, financial industry became capable of cutting service costs, providing more flexible time horizons, and less paperwork for its customers. But all these achievements made its business even more risky. Finance for most people is a black box. They do not understand what is happening although they are using it, they usually are not that well prepared for the risk associated with it. Even people working in the investment areas are not usually aware of what is waiting for them in the next phase of their behavior.

One famous case of people going directly into such a very visible but noisy destructive storms is the 2008 financial market problems, resulting in the whole global economy to face stagnation problems and financial sector to lose most of its credibility and very restrictive governmental regulation to be passed in most countries for financial sector [4], [5].

But what went wrong? How come nobody saw what was coming their way? Many more whys and how's are to be asked and for many more years to come scholars would be studying this phenomenon and the effect it has on our life and particularly on banking financial markets. Yet one thing is accepted within the context of study related to such phenomenon, we failed to predict the outcome correctly and thus the whole system went down to its crash margin. Thus, there is real need for reform in our prediction techniques regarding the risk associated with financial institution.

E-finance especially web-based finances including Emarkets for financial trades, such as E-banking, E-insurance, options, and securities, is subject to many scrutinization regarding its security, reliability, and sustainability. People especially in the lesser developed countries are suspicious of how reliable these new systems are. But customers are not the only one concerned with this kind of issues, regulatory offices

Omidreza Ghanadiof, Graduated of Industrial Management, School of Technology, University of Central Missouri, USA.

(e-mail: Omidof90@gmail.com) 
of governments worldwide are providing new guidelines and standards day in day out.

Still people with the most concern on risk regarding the Efinances are not the aforementioned customers and regulators; E-financers are the most concerned of all. First because they are dealing with these issues on daily routine and second because of the effect the loss of trust could have on the existence of their professional and corporate life.

Searching the net one can see a large number of firms and or people who lost their business and were ultimately omitted from market.[6] All these sorrowful failure stories are resulted from loss of trust. What all these people are searching for is a reliable prediction of the most probable futures, better stated a very reliable predictive risk assessment tool.[7] All kind of E-businesses and ultimately E-businesses such as Efinances are subject to very big trust ability question marks for the current and potential customers. Even a very small failure experience in monetary transaction due to some router malfunction might result in loss of business for a firm. Thus, anything that might go wrong is part of the total risk assessment E-finance provider should consider.

When dealing with risks we are either preventing or controlling it. In prevention we aim to stop preventable losses by the means of standardization, this is an area of interest especially for regulatory officials. Control is minimizing the undesired effect in the worst-case scenario. Risk management is prevention and control done in a simultaneous way. Assessing a risk, we aim at both preventive and controlling tasks so there is a greater need of prediction of what might go wrong and how.

Neural network has been found to be not just a soft computation methodology but as a matter of fact a new approach for handling problems that are not as formulate-able as we used to believe.

This new generation of computational method has proven to be able to handle problems in large scale while being able to undergo lesser amount of simplicity and maintaining its accuracy despite its youth and absence of a unified widely accepted convention system.

Neural networks are wide growing computational method and as a matter of fact they found their way into world of finance far sooner than expected due to the facts mentioned previously and their flexibility.

Here by flexibility, we mean that the network proposed for one application may find its way into applications that its creator may not have even imagined.

Marketing and finance Behavioral forecasting have been of interest since many years ago and recently it has grown to such large scale problem in which it may even be considered as one of the problems of modern societies, widely growing private business and etc. which requires a definite answer so they may attract larger amount of founding and so on.

\section{MULTILAYER NETWORK}

The multilayer feedforward neural network is the backbone of the Neural Network, and it has found its application for both function fitting and pattern recognition problems and with the addition of a tapped delay line, it can also be used for prediction problems. In this section we have tried to demonstrate how multilayer networks are a must for implementation of such network [8], [[9]. The workflow for the general neural network design process has seven primary steps:

Collect data, Create the network, Configure the network, Initialize the weights and biases, Train the network, Validate the network (post-training analysis), Use the network. Organization Objects, Data, and Training Styles presents stages $2 \& 3$, and reveal the basics of steps 5 and 7 . The forthcoming segments intend to exhibit stages 2-7 of the plan interaction for multi-facet organizations [10].

\section{Collect AND PREPARE THE DATA}

Prior to starting the organization configuration measure, you first gather and get ready example information. It is generally hard to join earlier information into a neural organization. Thusly the organization can only be pretty much as precise as the information that are utilized to prepare the organization.

It is significant that the information covers the scope of contributions for which the organization will be utilized. Multi-facet networks can be prepared to sum up well inside the scope of contributions for which they have been trained [3], [8]. Nonetheless, they cannot precisely extrapolate past this range. Therefore, it's important that the preparation and training data cover the full scope of the info space.

There are two steps that need to be performed before the data are used to train the network. First the data need to be preprocessed and divided into subsets. The next two sections describe these two steps [4], [12].

\section{A. Preprocessing and Post-processing}

Neural organization train can be done more productive in the specific way if they pay attention more to input and targets. (The most well-known of these are given automatically when you make a network, and they become some portion of the network object, so when data going to be used, the data will come to the network) [13]. In multi-facet organizations, sigmoid exchange functions are utilized in the secret layers [14], [5]. These capacities become basically immersed when the net information is more prominent than three $(\exp (-3) \cong 0.05)$. On the off chance that this occurs toward the start of the preparation interaction, the slopes will be little, and the organization training will be moderate [20]. The net input is a product of the input times the weight plus the bias in the first layer of the network. If the input is enormous, the weight should be very small to prevent the transfer function from becoming saturated. It is standard practice to normalize the contributions prior to applying them to the network. By and large, the standardization step is applied to both the info vectors and the objective vectors in the data set. Along these lines, the organization yield consistently falls into a standardized reach [2], [12]. It is most straightforward to consider the neural organization having a preprocessing block that shows up between the input and the principal layer of the organization and a post-handling block that shows up between the last layer of the organization and the yield [32]. 


\section{B. Dividing the Data}

When training multilayer networks, the general practice is to first divide the data into three subsets. The first subset is the training set, then the validation set, and create the network object.

\section{REPRESENTING UNKNOWN OR DON'T CARE TARGETS}

If a network has several outputs, some elements of any target vector may be known while others are unknown, but we do not want to have these targets to have an impact on training which can be represented with neural network values, but [10]. One solution is to remove the partially unknown target vector and its associated input vector from the training set. A better solution is to represent those unknown targets with neural network values [[9], [14].

\section{CReate, Configure, ANd Initialize the Network}

The multilayer feedforward network can be trained for function (nonlinear regression) or pattern recognition.

There are two distinct ways in which preparing can be carried out: steady mode and group mode. incremental mode and batch mode [24]. In incremental mode, the gradient is figured, and the loads are refreshed after each information is applied to the organization. In batch mode, every one of the contributions to the preparation set are applied to the organization before the loads are updated. For preparing multi-facet feedforward networks, any standard mathematical advancement calculation can be used to streamline the presentation work, yet there are a couple of key ones that have shown brilliant performance for neural organization preparation. These improvement techniques utilize either the slope of the network execution regarding the organization loads or the Jacobian of the organization mistakes with regard to the weights.

The gradient and the Jacobian are determined utilizing a procedure called the backpropagation calculation, which includes performing calculations in reverse through the organization. The backpropagation computation is determined utilizing the chain rule of math [22].

\section{TRAINING AlgORITHMS}

As an outline of how the preparation functions, consider the most straightforward enhancement calculation which is the gradient descent. It refreshes the organization loads and biases toward the path in which the exhibition function diminishes most quickly, the negative of the inclination. One cycle of this calculation can be written:

$\mathrm{X}_{\mathrm{K}+1}=\mathrm{X}_{\mathrm{K}}+\alpha_{\mathrm{K}} \mathrm{G}_{\mathrm{K}}$

where in (1) XK is a vector of current loads and biases, GK is the current gradient, and $\alpha \mathrm{K}$ is the learning rate. This condition is iterated until the organization combines [4]. As a note on wording, the expression "backpropagation" is once in a while used to allude explicitly to the gradient descent algorithm, when applied to neural organization preparing.
That phrasing isn't utilized here, since the way toward processing the slope and Jacobian by performing calculations in reverse through the organization is applied in the entirety of the training functions recorded previously. It is clearer to utilize the name of the particular improvement calculation that is being utilized, as opposed to utilize the term backpropagation alone [5]. Additionally, the multi-facet network is in some cases alluded to as a backpropagation network. In any case, the backpropagation method that is utilized to figure inclinations and Jacobians in a multi-facet network can additionally be applied to various organization models.

Appropriately prepared multi-facet networks will in general offer sensible responses when given sources of info that they have never seen. Commonly, another information prompts a precise yield, if the new info is similar to inputs utilized in the preparation set. This speculation property makes it conceivable to prepare a network on an agent set of information/target combines and get great outcomes without preparing the network on all conceivable

\section{PREDICTIVE NeURAL Network TOPOLOGY}

The multi-layer nonlinear predictive controller has the ability to look ahead if we are able to provide the network with just data so the data accusation and evaluation takes place with every epoch of calculation but in this network as the existence of transfer function in necessary and there for this network may not be implemented for cases where direct calculation is not possible but as we double the size of our network we gain the ability to calculate the input of our function side by side of the networks output there for by the use of this network we are able to regenerate another input function that yields wrong results or better to say unfit results [7], [10].Out of the results obtained from the regeneration of input function we are able to compute the error in our assessment and predict the time in which our system shall experience failure and the frequently of reoccurrence of this failure [24], [10]. The error is the difference between the fake input function and the actual output function that is resulted from direct calculation and obtained just after the weight function is tuned as best as possible. So, the fake new input function yields the fact that how much we are off the facts. This means that the market behavior is far off our prediction so we are to re-justify our weight function for which they may not be the optimal values, but they are values that prevent the crossover of the fake input function [22].

The number of times that such cross over happens yields the number of times we are going to experience failure i.e., after 20 years we shall experience failure three times in Fig. 1.

The model represented in figure 1 is an ideal case of decision making for the case where sub-linear behaviors can be considered [20], [12]. Fig. 2 represents the final stages of training the network and represents the behavior of the network when feed with statics and data that is proportional to the number of expected variables, and they may be approximated by gaps of behavior. 


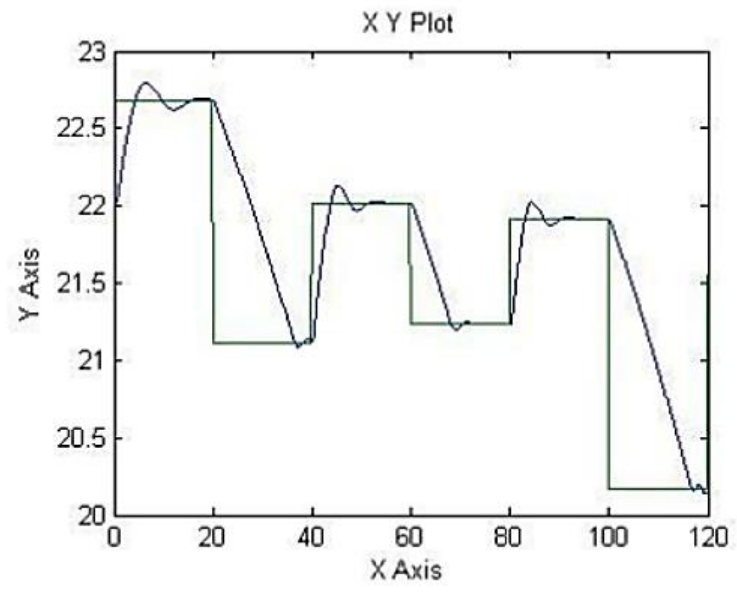

Fig. 1. System uncertainty template in accordance with its fake input and its calculated output behavior.

\section{CONCLUSION}

Regardless of the type of risks we are dealing with, from risk of malfunctions to insider's threat, we are dealing with risks which are multilayered in nature and usually we cannot approximate them by linear models. Our understanding of risks is evolving and developing every day [22]. Thus, the need for a new model of prediction is of importance.

This network has the ability to predict the outcome of certain policy makings before they see action by comparing the error that shall occur due to those policies that is the fake input function that has been mentioned and compering these results to the actual behavior of the systems input. This process for our simple model is shown in Fig. 3.

Future studies could be done using this predictive neural network model for any kind of risk assessment, but this predictive model is not limited to risk assessment, and it might be used for any kind of control in uncertain situation risk - in future we aim to apply this model to different aspect of financial risk not just E-finance.
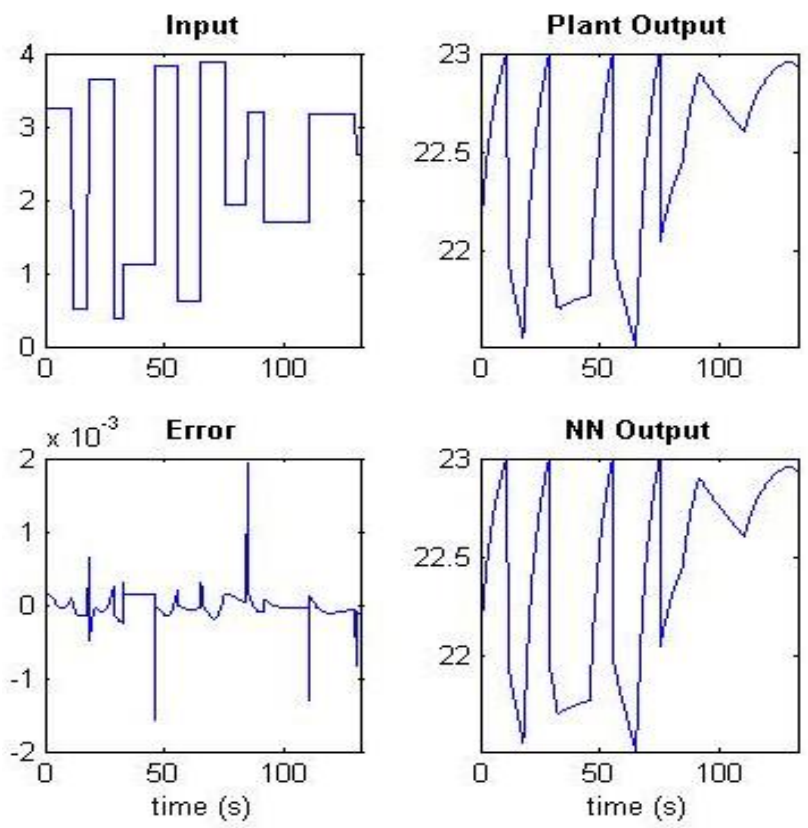

Fig. 2. Systems behavior when feed with the training data.
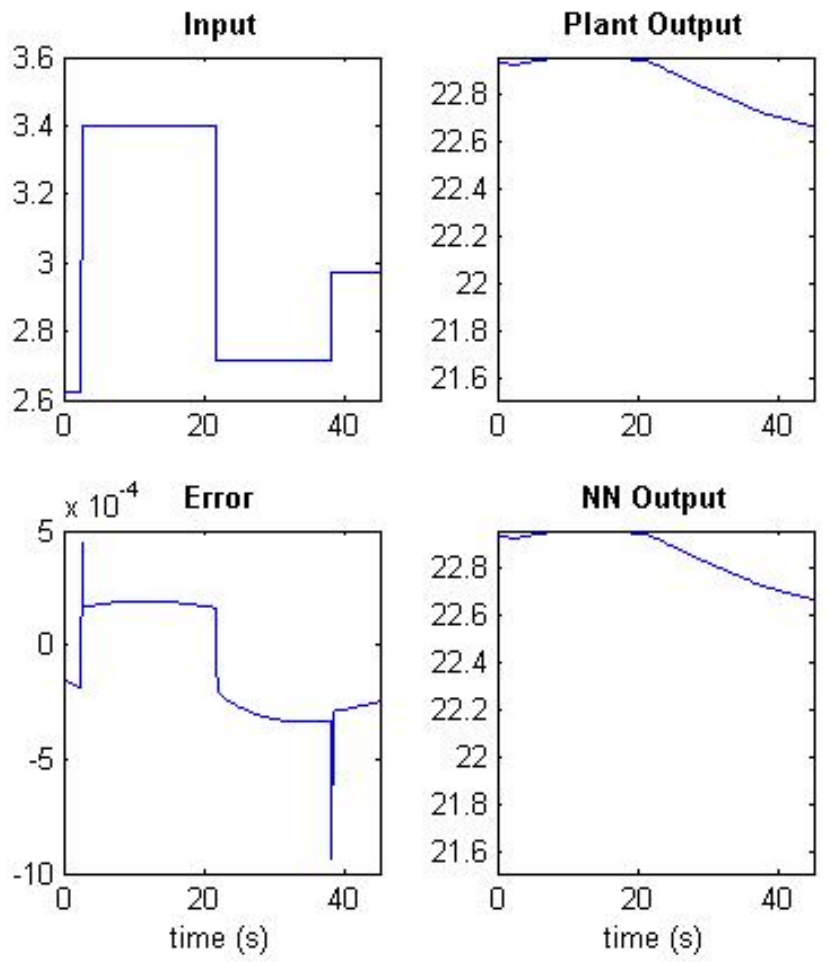

Fig. 3. Systems behavior when tuning its weight function according to its fake input function in the first epochs of calculation.

\section{REFERENCES}

[1] Bowersox, D. J., Closs, D., \& Helferich, O. K. (1986). Logistics management. New York: Macmillan.

[2] P. Campolucci, A. Uncini, F. Piazza, B.D. Rao, On-line learning algorithms for locally recurrent neural networks, IEEE Transactions on Neural Networks 10 (2) (1999) 253-271.

[3] Zadeh, L. A. (1973). Outline of a new approach to the analysis of complex systems and decision processes. IEEE Transactions on Systems, Man, and Cybernetics, SMC-3(1), 28 - 44.

[4] S. Nolfi, D. Floreano, Evolutionary Robotics, MIT Press, 2000.

[5] D. Hein, M. Hild, R. Berger, Evolution of biped walking using neural oscillators and physical simulation, in: U. Visser, et al.et al. (Eds.), RoboCup 2007, vol.5001, LNAI, 2008, pp. 433-440.

[6] Mohajerani, P., \& Miremadi, A. (2013). Exploring two main perspectives towards customer satisfaction in hotel industry: managers and customers. International Journal of Academic Research in Business and Social Sciences, 3(9), 245.

[7] Ghanadiof, O., Sanayei, A., \& Emami, M. (2021). Effect of Customer Perception on Salesperson Owned Commitment in CustomerSalesperson Relationship. European Journal of Business \& Management Research. https://doi.org/10.24018/ejbmr.2021.6.4.964

[8] Oh S-K, Pedrycz W, Park H-S. Rule-based Multi-FNN identification with the aid of evolutionary fuzzy granulation. J Knowl-Based Syst 2004:17(1):1 - 13 .

[9] Cordon O, Gomide F, Herrera F, Hoffmann F, Magdalena L. Ten years of genetic fuzzy systems: current framework and new trends. Fuzzy Sets Syst 2004;141(1):5 - 31 .

[10] Fardhosseini, M. S., Soltaninejad, M., Karji, A., Ghorbani, Z. \& Ghanadiof, O. (2021). Qualitative Evaluation of 5S Application Considering the Experience of Electrical Construction Experts. American Journal of Applied Sciences, 18(1), 51-60. https://doi.org/10.3844/ajassp.2021.51.60.

[11] S.W. Wilson, State of XCS classifier system research, Second International Workshop on Learning Classifier Systems during GECCO99, 1999.

[12] Yeh, C. C., Chi, D. J., \& Hsu, M. F. (2010). A hybrid approach of DEA, rough set and support vector machines for business failure prediction. Expert Systems with Applications, 37, 1535-1541.

[13] Miremadi, A., \& Ghanadiof, O. (2021). The Ultimate Influences of Brand Equity Dimensions on Consumer Decision in Hi-Tech Market. Academic Journal of Research and Scientific Publishing. doi. org/10.52132/Ajrsp.e. 
[14] A. Campo, J. Santos, Evolution of adaptive center-crossing continuous time recurrent neural networks for biped robot control, in: Proceedings European Symposium on Artificial Neural Networks (ESANN 2010), Bruges (Belgium),2010, pp. 535-540.

[15] A.S. Weigend, Time series analysis and prediction using gated experts with application to energy demand forecast, Applied Artificial Intelligence 10 (1996) 583-624.

[16] X. Yao, Evolving articular neural networks, Proceedings IEEE 87 (1999) 1423-1447.

[17] Oh S-K, Pedrycz W, Park H-S. Hybrid identification in fuzzy-neural networks. Fuzzy Sets Syst 2003;138(2):399 - 426.

[18] R.A. Jacobs, M.I. Jordan, S.J. Nowlan, G.E. Hinton, Adaptive mixtures of local experts, Neural Computation 3 (1991) 79-87.

[19] R. Beer, Parameter space structure of continuous recurrent neural networks, Neural Computer. 18 (2006) 3009-3051.

[20] Miremadi, A., Kenar Roudi, J., \& Ghanadiof, O. (2021). Evaluation on Role of Electronic Word of Mouth (EWOM) Ads in Customers' Emotions and Choices in E-Shops. International Journal of Industria Marketing, 6(1). https://doi.org/10.5296/ijim.v6i1.

[21] Cherkassky V, Gehring D, Mulier F. Comparison of adaptive methods for function estimation from samples. IEEE Trans Neural Netw 1996; 7:969-84.

[22] Miremadi, A., Golchobian, M. M. A., \& Ghanadiof, O. (2021). Requirement and Architecture of Organization Development. European Journal of Business and Management Research, 6(4), 55-64. https://doi.org/10.24018/ejbmr.2021.6.4.932.

[23] Gupta, M. M., \& Qi, J. (1991). On fuzzy neuron models. Proceedings of international joint conference on neural networks, Seattle, II, 43124436.

[24] Miremadi, A., \& Ghanadiof, O. (2021). CRM Competitive Strategy in Financial Institutions. European Journal of Business and Management Research, 6(3), 111-117. https://doi.org/10.24018/ejbmr.2021.6.3.867.

[25] de Jager, C. P. C., van Wijk, P. T. L., Mathoera, R. B., de JonghLeuvenink, J., van der Poll, T., \& Wever, P. C. (2010). Lymphocytopenia and neutrophil-lymphocyte count ratio predict bacteriemia better than conventional infection markers in an emergency care unit. Critical Care, 14(R192), 108-115.

[26] Miremadi, A., \& Khoei, R. (2013). The art of visual merchandising on consumer buying behavior. International Journal Contemporary Business Studies, 4(6), 34-50.

[27] L.R. Rabiner, B.H. Juang, An introduction to Hidden Markov models, IEEE ASSP Magazine3 (1) (1986) 4-16.

[28] S. Wischmann, F. Pasemann, From passive to active dynamic 3D bipedal walking - an evolutionary approach, in: M. Armada, P. Gonzalez de Santos (Eds.), Climbing and Walking Robots: Proceedings of the 7th International Conference (CLAWAR 2004), SpringerVerlag, 2004, pp. 737-744.

[29] G. McHale, P. Husbands, GasNets and other evolvable neural networks applied to bipedal locomotion, in: From Animals to Animats 8Proceedings of the Eighth International Conference on the Simulation of Adaptive Behavior, 2004, pp. 163-172.

[30] Ghanadiof, O. (2021). Customer loyalty and powerful brand in heavy machinery industry. European Journal of Business and Management Research, 6(3). https://doi.org/10.24018/ejbmr.2021.6.3.903.

[31] Handfield, R. B., \& Nichols, E. L., Jr (1999). Introduction to supply chain management. Englewood Cliffs, NJ: Prentice Hall. 